\title{
Long-term follow-up study of patients with adrenal incidentalomas
}

\author{
Rossella Libè ${ }^{1}$, Chiara Dall'Asta ${ }^{1}$, Laura Barbetta ${ }^{1}$, Andrea Baccarelli ${ }^{2}$, Paolo Beck-Peccoz ${ }^{1}$ and Bruno Ambrosi ${ }^{1,3}$ \\ ${ }^{1}$ Institute of Endocrine Sciences, University of Milan, Ospedale Maggiore IRCCS, Milan, Italy, \\ ${ }^{2}$ Genetic Epidemiology Branch, Division of Cancer Epidemiology and Genetics, National Cancer Institute/NIH, Bethesda, Maryland, USA and \\ ${ }^{3}$ Division of Endocrinology and Diabetology, Istituto Policlinico San Donato, San Donato Milanese, Milan, Italy \\ (Correspondence should be addressed to B Ambrosi, Istituto Policlinico San Donato, via Morandi 30, 20097 San Donato Milanese, Milan, Italy; Email: \\ bruno.ambrosi@unimi.it)
}

\begin{abstract}
Background: The incidence of adrenal incidentalomas has sharply increased in recent decades and concurrent subtle endocrine abnormalities, or even subclinical conditions, have been identified. Nonetheless, data concerning possible changes in adrenal size and/or hormonal pattern during follow-up are still inadequate.

Objective: To evaluate long-term morphological and functional evolution of adrenal incidentalomas after initial diagnosis and to identify possible risk factors for hormonal hyperactivity and mass enlargement.

Patients: Sixty-four patients (34-79 years) were followed-up for 12-120 months (median 25.5 months). Initial computerized tomography scan showed a unilateral mass in 51 patients and bilateral lesions in 13 patients. Average mass diameter at diagnosis was $2.5 \pm 0.1 \mathrm{~cm}$ (range 1.0-4.0). Twelve patients had subclinical Cushing's syndrome, 41 had mild hormonal alterations, and 11 had normal adrenal function at baseline. All patients were investigated by morphological and functional evaluation 6 and 12 months after diagnosis, and then at 1-year intervals.

Results: During follow-up, a mass size increase $\geq 1 \mathrm{~cm}$ was observed in 13 patients, and 18 developed further subtle endocrine alterations. Cumulative risk of developing endocrine abnormalities was $17 \%$ at 1 year, $29 \%$ at 2 years, and $47 \%$ at 5 years. The risk was higher in the first 2 years of follow-up if the initial tumor diameter was $\geq 3 \mathrm{~cm}$. Overall, cumulative risk of mass enlargement was $6 \%$ at 1 year, $14 \%$ at 2 years, and $29 \%$ at 5 years, and it was greater in patients with normal adrenal function than in those with subtle hormonal abnormalities $(P<0.05)$. One female subject showed a mass enlargement after 6 months of follow-up and was eventually diagnosed with non-Hodgkin's lymphoma.

Conclusions: Patients with an adrenal incidentaloma are at risk for tumor growth and development of hormonal alterations. The risk of adrenal malignancy, although not elevated, also indicates the need for long-term follow-up.
\end{abstract}

European Journal of Endocrinology 147 489-494

\section{Introduction}

Adrenal incidentalomas are masses of $1 \mathrm{~cm}$ or more in diameter, discovered incidentally during imaging investigation of non-adrenal disorders. This clinical condition is frequently diagnosed and its prevalence ranges between 1 and $5 \%$ in different series (1-3). Adrenal incidentaloma has been associated with subtle endocrine abnormalities, such as low dehydroepiandrosterone sulfate (DHEA-S) levels, and increased 17-hydroxyprogesterone (17-OHP) and 11-deoxycortisol responses to adrenocorticotropin (ACTH) administration (4-9). Silent pheochromocytomas and subclinical Cushing's syndrome (SCS), which is characterized by autonomous cortisol-secreting adenomas without signs or symptoms of hypercortisolism, were also found, albeit less frequently $(9-13)$.

The type and the frequency of endocrine and imaging investigations to be performed during followup remain controversial, because data on possible adrenal size increase and hormonal pattern changes over time are still inadequate. The incidence of either mass enlargement or hormonal alterations during long-term follow-up has not been thoroughly investigated. Recently, Barzon et al. (14) evaluated long-term clinical, hormonal, and morphological outcomes in patients with adrenal incidentalomas in order to obtain prognostic factors for disease progression. 
The aim of this retrospective study was to evaluate the cumulative risk of developing endocrine abnormalities and of mass enlargement, as detected by computerized tomography (CT), in a group of patients with incidentalomas with a minimum follow-up of 12 months.

\section{Subjects and methods}

Sixty-four patients (forty females, median age $=61$ years, range 39-79; twenty-four males, median age 59.5 years, range 34-76) were followed up for 12-120 months (median $=25.5$ months). Adrenal masses were discovered incidentally through imaging techniques performed for the evaluation of unrelated disorders. CT scan demonstrated a unilateral mass in fifty-one patients (thirty-two in the right gland and nineteen in the left), and bilateral lesions in thirteen, the mean diameter being $2.5 \pm 0.1 \mathrm{~cm}$ (range 1.0-4.0). Thirty-eight patients had a lesion $<3 \mathrm{~cm}$, and twentysix $\geq 3 \mathrm{~cm}$. In forty-two patients ultrasonography (US) was also performed: US mass size was concordant with CT measurement in only $55 \%$ of cases. In these patients, the mean diameter was $26.7 \pm 1.6 \mathrm{~mm}$ at US, and $26.8 \pm 1.4 \mathrm{~mm}$ at CT scan. A close correlation was found between US and CT measurements of mass size $(r=0.65, P<0.0001)$. Hypertension was present in $55 \%$ of the cases, overt type- 2 diabetes mellitus in 22\%, and overweight in 31\%. During follow-up, additional patients developed high blood pressure $(n=4)$, type- 2 diabetes mellitus $(n=1)$, and overweight $(n=10)$. Three of these patients showed a concurrent mass enlargement $\geq 1 \mathrm{~cm}$.

The following hormonal determinations were performed in all patients by commercial kits, as previously reported (6): plasma ACTH and serum cortisol, 17-OHP, DHEA-S, $\Delta 4$-androstenedione and testosterone levels, plasma renin activity and aldosterone in supine and upright positions, 24-h urinary free cortisol (UFC) and catecholamine excretion. Serum cortisol and 17-OHP levels were also measured before and after exogenous ACTH injection $(250 \mu \mathrm{g}$ i.v.). ACTH and cortisol levels were also evaluated after an overnight $1 \mathrm{mg}$ dexamethasone test. In patients with SCS, cortico-

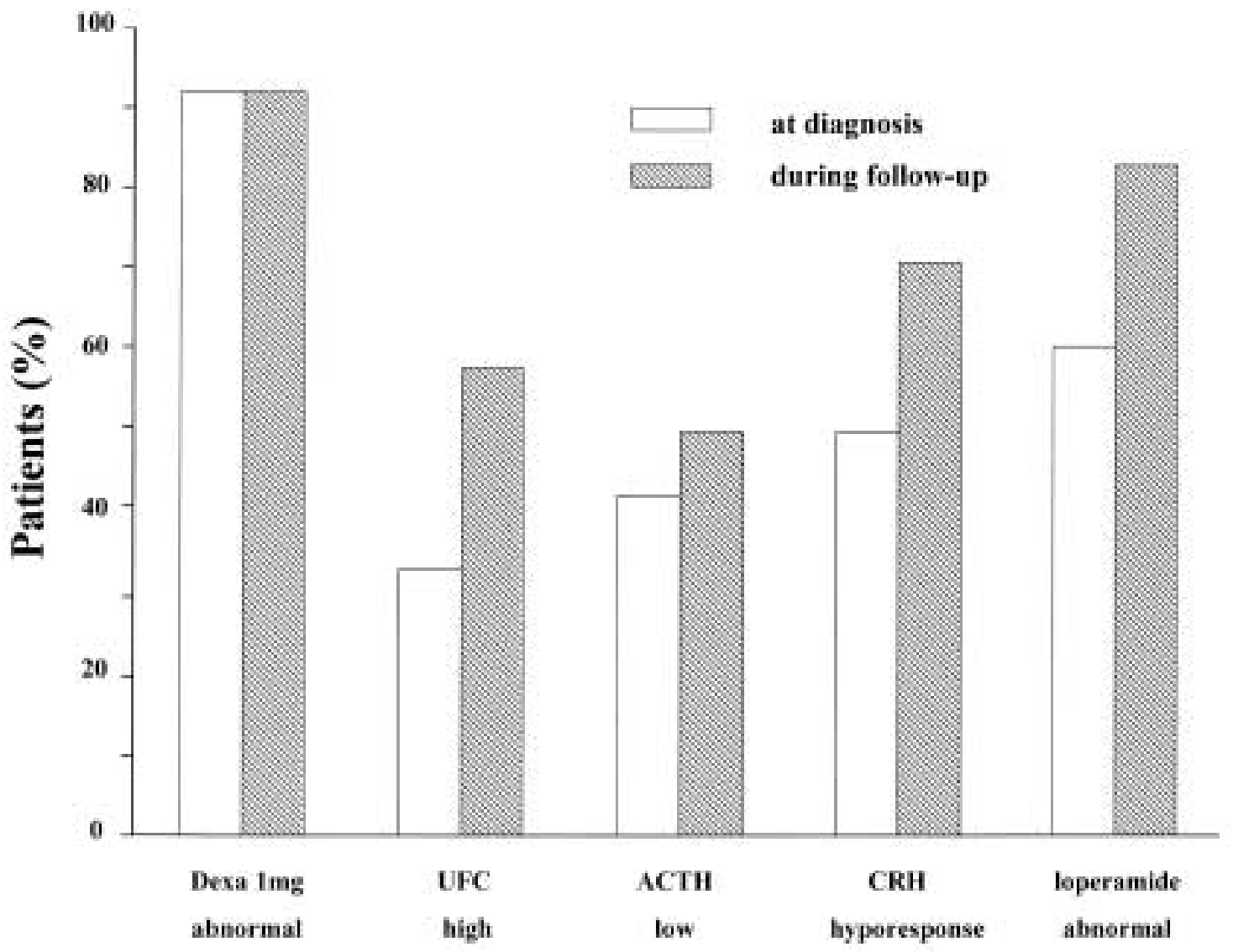

Figure 1 Frequency of hormonal abnormalities in 12 patients with subclinical Cushing's syndrome (group A) at first presentation and during follow-up, in both basal and dynamic conditions. Dexa, dexamethasone; UFC, urinary free cortisol, ACTH, adrenocorticotropin; $\mathrm{CRH}$, corticotropin-releasing hormone. 
tropin-releasing hormone (CRH; $1 \mu \mathrm{g} / \mathrm{kg}$ i.v.) and loperamide tests (16 mg orally) were also performed.

As previously reported $(6,9,10)$, SCS was defined by the absence of signs or symptoms of hypercortisolism and by the presence of at least two of the following criteria: inadequate cortisol inhibition after $1 \mathrm{mg}$ dexamethasone (cortisol levels $\geq 138 \mathrm{nmol} / \mathrm{l}$ ), high or high-normal UFC excretion ( $\geq 275 \mathrm{nmol} / 24 \mathrm{~h})$, low or low-normal plasma ACTH levels $(<0.7 \mathrm{pmol} / \mathrm{l})$, blunted ACTH increase after $\mathrm{CRH}$ test $(<4.4 \mathrm{pmol} / \mathrm{l})$, and lack of cortisol suppression after opioid agonist loperamide administration ( $\geq 138 \mathrm{nmol} / \mathrm{l}$ ).

The patients were reinvestigated at 6 and 12 months and then yearly, by clinical examination, routine chemistry, hormonal determinations in basal and dynamic conditions, and by CT scan. During the follow-up the mass was defined as enlarged when the increase was equal or more than $1 \mathrm{~cm}$.

\section{Statistical analysis}

The Kaplan-Meier method was used to estimate the cumulative risk of endocrine abnormalities and mass enlargement during follow-up. The Wilcoxon (Breslow) test for equality of survivor functions was used for group comparisons. The Bonferroni correction for multiple comparisons was implemented, if needed. We used simple regression analysis to assess betweenvariable correlation. $P$ values $<0.05$ were considered significant.

\section{Results}

At diagnosis, twelve patients had SCS (group A): eleven out of twelve patients showed a lack of cortisol suppression after dexamethasone, four out of twelve had high UFC, five out of twelve had low plasma ACTH levels, and three out of six had blunted ACTH responses to $\mathrm{CRH}$. Inadequate inhibition to opioidagonist loperamide was found in six out of ten cases (Fig. 1). In addition, nine patients had low DHEA-S levels, and eleven showed 17-OHP hyper-response to ACTH stimulation (peak $\geq 15 \mathrm{nmol} / \mathrm{l}$ ). Fifty-two patients had a non-functioning adrenal mass. Fortyone of them showed mild hormonal alterations (group B): high 17-OHP peak after ACTH test was found in thirty-four patients, DHEA-S levels were low in twenty-nine patients, UFC excretion was high in two cases, and plasma ACTH was low in two additional cases. One patient showed inadequate cortisol suppression after $1 \mathrm{mg}$ dexamethasone. No endocrine abnormalities were detected in the remaining eleven patients with a non-functioning adrenal mass (group C).

SCS persisted throughout the follow-up in all twelve group A patients: a lack of cortisol suppression after dexamethasone was confirmed in eleven cases, increased UFC excretion in seven and low basal ACTH in six cases; we found blunted ACTH responses to $\mathrm{CRH}$ in five out of seven cases, and inadequate inhibition to loperamide in five out of six cases

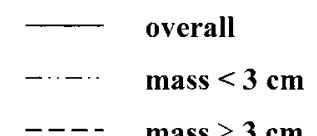

Figure 2 Estimated cumulative risk of developing endocrine abnormalities according with the mass size ( $<$ or $\geq 3 \mathrm{~cm})$ in patients with adrenal incidentalomas. 
(Fig. 1). None of the patients developed overt Cushing's syndrome. Twelve out of forty-one patients with mild hormonal alterations subsequently developed further endocrine abnormalities, but no subject developed SCS. In particular, a high 17-OHP peak after ACTH test was found in one patient, DHEA-S levels were low in three patients, plasma ACTH was decreased in four other cases and a lack of cortisol suppression after $1 \mathrm{mg}$ dexamethasone was observed in four patients. Moreover, DHEA-S levels were low in three of the eleven patients with normal adrenal function at diagnosis.

In order to estimate the cumulative risk of developing endocrine abnormalities, Kaplan-Meier curves were created. Data after 5 years were not analyzed owing to the small number of patients. When the whole group of patients was considered, the risk of developing additional endocrine abnormalities was $17 \%$ at 1 year, $29 \%$ at 2 years, $39 \%$ at 3 years, $39 \%$ at 4 years, and $47 \%$ at 5 years. When subjects were divided by mass size at diagnosis, the risk of further endocrine changes in the first 2 years of follow-up was higher in patients with mass size $\geq 3 \mathrm{~cm}$ than in those with mass size $<3 \mathrm{~cm}(23 \%$ vs $13 \%$ at 1 year, and $39 \%$ vs $24 \%$ at 2 years respectively). However, no overall significant difference between risk-curves was observed (Fig. 2).

As far as the mass size is concerned, lesion diameter at initial presentation was $<3 \mathrm{~cm}$ in thirty-eight patients and $\geq 3 \mathrm{~cm}$ in twenty-six patients. Group A patients had a larger mass size though than groups $\mathrm{B}$ and $\mathrm{C}(2.8 \pm 1.0,2.4 \pm 0.9$, and $2.4 \pm 0.9 \mathrm{~cm}$ respectively), but these differences were not statistically significant. Morphological follow-up by CT scan showed the presence of adrenal mass enlargement $\geq 1 \mathrm{~cm}$ in thirteen cases $(20 \%)$, four of whom had SCS. The average size increment was $1.7 \pm 0.2 \mathrm{~cm}$. No patient showed a tumor reduction or disappearance during follow-up.

When all patients were considered, cumulative risk of developing mass enlargement (i.e. diameter increase $\geq 1 \mathrm{~cm}$ ) was $6 \%$ at 1 year, $14 \%$ at 2 years, $17 \%$ at 3 years, $22 \%$ at 4 years, and $29 \%$ at 5 years. Figure 3 shows the risk of mass enlargement in patients with SCS, subtle endocrine abnormalities, or without any hormonal alteration. In group A (SCS) patients, the cumulative risk was $8 \%$ at 1 year, $31 \%$ at 2 years, and then remained stable up to 5 years. In group B patients, the risk was low in the first 2 years and increased afterward: $2.5 \%$ at 1 year, $2.5 \%$ at 2 years, $8 \%$ at 3 years, $16 \%$ at 4 years, and $33 \%$ at 5 years. As in group A, the risk increased in the eleven cases with normal adrenal function (group C) only in the first 2 years after diagnosis $(18 \%$ after 1 year, and $41 \%$ after 2 years) and thereafter remained stable. It is worth noting that one female subject exhibited a conspicuous tumor growth (from 1.5 to $4 \mathrm{~cm}$ ) 6 months after the first presentation resulting from a malignant lesion, diagnosed after surgery as a diffuse large B-cell non-Hodgkin's lymphoma.

Risk of mass enlargement was significantly lower in subjects with subtle abnormalities (group B) than in patients with normal adrenal function (group $\mathrm{C}$ ) $(P=0.03)$. Risk differences between subjects with SCS and subjects with mild endocrine abnormalities were not statistically significant $(P=0.35)$, possibly

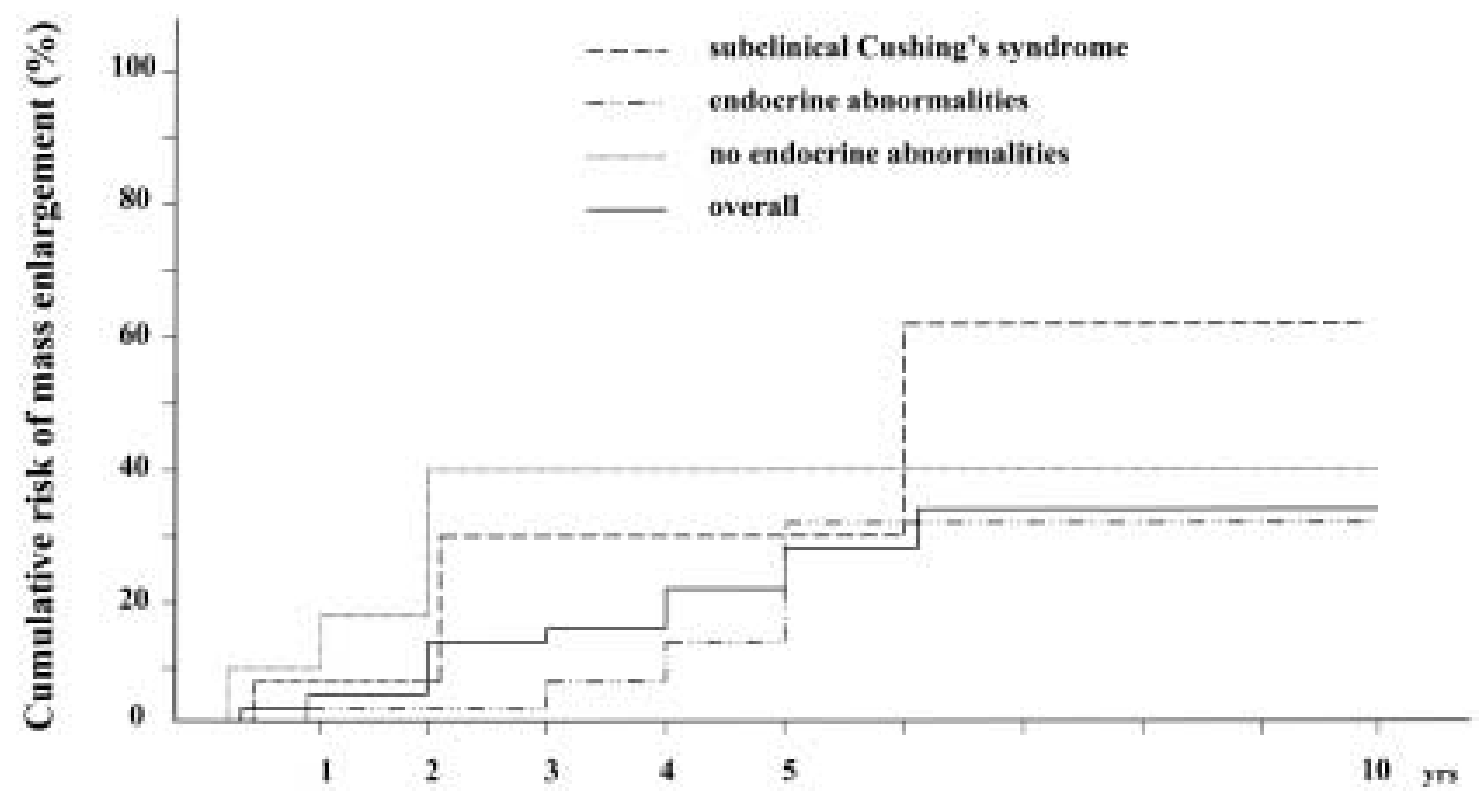

Figure 3 Estimated cumulative risk of showing adrenal mass enlargement in all patients with adrenal incidentalomas and in three groups according to endocrine data at presentation. There is a significant difference $(P<0.05)$ between the curves of groups $\mathrm{B}$ (endocrine abnormalities) vs C (no endocrine abnormalities). 
because of the small number of subjects included in the analysis.

During follow-up, nine patients underwent adrenalectomy. Seven of them, with a median follow-up of 30 months and including two subjects with SCS, had shown a substantial mass enlargement (mean tumor diameter from $2.7 \pm 0.4$ to $4.2 \pm 0.1 \mathrm{~cm}$ ). Surgery was performed in the remaining two cases (mean tumor diameter $=3.5 \mathrm{~cm}$ ) and also taking the patients' considerations into account. Histopathological examination revealed adrenocortical adenoma in six cases, myelolipoma, leiomyoma, and B-cell non-Hodgkin's lymphoma in the three remaining subjects.

\section{Discussion}

In the present study, we evaluated the risk of endocrine morbidity during follow-up of patients with an adrenal incidentaloma. Newly diagnosed subtle endocrine alterations appeared in $28 \%$ of the patients, most of them featuring a decrease in DHEA-S and ACTH levels, as previously reported $(14,15)$. Similar small changes in hormonal function have been reported by Barzon et al. (14) and Grossrubatscher et al. (15). Diagnosis of SCS was confirmed during follow-up, as endocrine abnormalities tended to become more evident. In fact, this condition encompasses a continuous spectrum of abnormalities, ranging from mild functional alterations in the hypothalamic-pituitaryadrenal axis towards the appearance of overt adrenal Cushing's syndrome $(14,16)$. However, in our retrospective study no patient showed an evolution to overt clinical hypercortisolism, in agreement with previous reports $(11,15)$. When the initial mass size was considered as a risk factor for adrenal hyperfunction, patients with a mass diameter $\geq 3 \mathrm{~cm}$ showed a higher cumulative risk of developing hormonal abnormalities than those with smaller lesions. The risk was confined to the first 2 years of follow-up, indicating the need for hormonal evaluation during this time-period.

The second issue addressed in the present study concerned the risk of mass enlargement. Most patients did not show any change in mass size during follow-up. We found an enlargement $\geq 1 \mathrm{~cm}$ in $20 \%$ of the cases, similar to two previous investigations $(14,15)$ that reported an increase in tumor size in $17 \%$ and $15 \%$ of patients respectively. No mass enlargement was observed in two other small studies $(17,18)$ while, in larger series, tumor growth was detected in a very low percentage of cases $(3.3-4.3 \%)(19,20)$. However, in one of these studies (20), the investigators obtained only indirect data from office records, or by telephone or mail contacts for most patients. Previous studies have documented occasional reduction or disappearance of the adrenal lesions $(14,15)$, a finding not confirmed in the present study.
We observed tumor growth in patients with subclinical hypercortisolism, as well as in those with minor endocrine abnormalities. In the former group, however, tumor growth occurred early after diagnosis and was slightly more frequent than in the latter. The possible role in tumor growth of a higher intra-adrenal steroidogenic activity, driven by ACTH or other factors, remains to be elucidated. A similar cumulative risk of mass increase in subjects with subtle endocrine abnormalities was also reported by Barzon et al. (14), while Grossrubatscher et al. (15) did not find any difference in mass enlargement between patients with or without 17-OHP hyper-response to ACTH stimulation. Some patients with normal hormonal function also showed mass enlargement and, in this respect, the possible risk of malignant transformation must always be considered. Many studies have suggested that the likelihood of malignant transformation at long-term follow-up is very small $(14,17,19,20)$. We observed a conspicuous mass increase in one woman 6 months after diagnosis: adrenalectomy was performed to remove the lesion, which was identified as a primary non-Hodgkin's lymphoma. In recent years, the existence of this rare disease has been increasingly reported thanks to advanced diagnostic imaging techniques (21).

On the whole, these data indicate that conservative management may be appropriate in most patients with adrenal incidentalomas, since mass size did not change in $80 \%$ of the cases. However, a prolonged follow-up should be recommended, independently of the mass size and the hormonal pattern at first presentation. CT scans, although a close correlation with US results was found, should be repeated every 6 months for at least 2 years and then yearly, in order to either detect malignant transformation or indicate, when the mass diameter becomes larger than $4 \mathrm{~cm}$, the necessity of adrenalectomy, as occurred in seven of our patients.

In conclusion, the present results suggest that long-term follow-up of adrenal incidentaloma is recommended, given the possibility of progressive tumor growth or the evolution to hormonal hypersecretion. Although the risk of malignant transformation is very low, careful management with morphological evaluation and hormonal determination is appropriate.

\section{Acknowledgements}

We thank Antonia Maffini for her technical assistance.

\section{References}

1 Kloos RT, Gross MD, Francis IR, Korobkin M \& Shapiro B. Incidentally discovered adrenal masses. Endocrine Reviews 199516 460-484.

2 Gross MD \& Shapiro B. Clinically silent adrenal masses. Journal of Clinical Endocrinology and Metabolism 1993 77 885-888. 
3 Abecassis M, McLoughin MJ, Langer B \& Kudlow JE. Serendipitous adrenal masses: prevalence, significance and management. American Journal of Surgery $1985149783-788$.

4 Seppel T \& Schlaghecke R. Augmented $17 \alpha$-hydroxyprogesterone response to ACTH stimulation as evidence of decreased 21-hydroxylase activity in patients with incidentally discovered adrenal tumour ('incidentalomas'). Clinical Endocrinology 1994 $41445-451$.

5 Osella G, Terzolo M, Borretta G, Magro G, Alì A, Piovesan A et al. Endocrine evaluation of incidentally discovered adrenal masses (incidentalomas). Journal of Clinical Endocrinology and Metabolism $1994791532-1539$.

6 Ambrosi B, Peverelli S, Passini E, Re T, Ferrario R, Colombo P et al. Abnormalities of endocrine function in patients with clinically 'silent' adrenal masses. European Journal of Endocrinology 1995 $132422-428$.

7 Sadoul JL, Kézachian B, Altare S, Hadjali Y \& Canivet B. Apparent activities of 21-hydroxylase, $17 \alpha$-hydroxylase and 17,20 lyase are impaired in adrenal incidentalomas. European Journal of Endocrinology $2000141238-245$.

8 Tòth M, Ràcz K, Adleff V, Varga I, Fùto L, Jarab C et al. Comparative analysis of plasma 17-hydroxyprogesterone and cortisol responses to ACTH in patients with various adrenal tumors before and after unilateral adrenalectomy. Journal of Endocrinological Investigation 200023 287-294.

9 Mantero F, Terzolo M, Arnaldi G, Osella G, Masini AM, Alì A et al. A survey on adrenal incidentaloma in Italy. Journal of Clinical Endocrinology and Metabolism 200085 637-644.

10 Reincke M, Nieke J, Krestin GP, Saeger W, Allolio B \& Winkelmann W. Preclinical Cushing's syndrome in adrenal 'incidentalomas': comparison with adrenal Cushing's syndrome. Journal of Clinical Endocrinology and Metabolism 199275 826-832.

11 Terzolo M, Osella G, Alì A, Borretta G, Cesario F, Paccotti P et al. Subclinical Cushing's syndrome in adrenal incidentaloma. Clinical Endocrinology $1998 \mathbf{4 8} 89-97$.

12 Mantero F \& Arnaldi G. Management approaches to adrenal incidentalomas. Endocrinology and Metabolism Clinics of North America 200029 107-125.
13 Reincke M. Subclinical Cushing's syndrome. Endocrinology and Metabolism Clinics of North America 200029 43-56.

14 Barzon L, Scaroni C, Sonino N, Fallo F, Paoletta A \& Boscaro M. Risk factors and long-term follow-up of adrenal incidentalomas. Journal of Clinical Endocrinology and Metabolism $1999 \mathbf{8 4}$ $520-526$.

15 Grossrubatscher E, Vignati F, Possa M \& Loli P. The natural history of incidentally discovered adrenocortical adenomas: a retrospective evaluation. Journal of Endocrinological Investigation $200124540-550$

16 Hensen J, Buhl M, Bähr V \& Oelkers W. Endocrine activity of the 'silent' adrenocortical adenoma is uncovered by response to corticotropin-releasing-hormone. Klinische Wochenschrift $1990 \mathbf{6 8}$ 608-614.

17 Virkkala A, Valimaki M, Pelkonen R, Huikuri K, Kahri A, Kivisaari L et al. Endocrine abnormalities in patients with adrenal tumours incidentally discovered on computed tomography. Acta Endocrinologica $198912167-72$.

18 Sirén J, Tervahartiala P, Sivula A \& Haapiainen R. The natural course of adrenal incidentalomas: a 7 year follow-up study. World Journal of Surgery $1998 \mathbf{2 4} 579-582$.

19 Bastounis EA, Karayiannakis AJ, Anapliotou ML, Nakopoulou L, Makri GG \& Papalambros EL. Incidentalomas of the adrenal gland: diagnostic and therapeutic implications. The American Surgeon 199763 356-360.

20 Barry MK, van Heerden JA, Farley DR, Grant CS, Thompson GB \& Ilstrup DM. Can adrenal incidentalomas be safely observed? World Journal of Surgery 199822 599-604.

21 Salvatore JR \& Ross RS. Primary bilateral adrenal lymphoma. Leukemia and Lymphoma 199934 111-117.

Received 17 December 2001

Accepted 4 June 2002 